Article

\title{
An Improvement Differential Evolution Algorithms for the Crop Planning in the Northeastern Region of Thailand
}

Udompong Ketsripongsa ${ }^{1}$, Rapeepan Pitakaso ${ }^{2}$, Kanchana Sethanan ${ }^{3}$ and Tassin Srivarapongse 4

1 Metaheuristics for Logistics Optimization Laboratory, Department of Industrial Engineering, Ubon Ratchathani University, Ubon Ratchathani, Thailand, Email: udompong.jo@gmail.com

2 Metaheuristics for Logistics Optimization Laboratory, Department of Industrial Engineering, Ubon Ratchathani University, Ubon Ratchathani, Thailand, Email: rapeepan.p@ubu.ac.th

3 Department of Industrial Engineering, Faculty of Engineering, Khon Kaen University, Khon Kaen, Thailand, Email: skanch@kku.ac.th

4 Department of Economics, Faculty of Business Administration, Rajamangala University of Technology Thanyaburi, Patumthani, Thailand, Email: tassin66@hotmail.com

* Correspondence: rapeepan.p@ubu.ac.th; Tel.: +66-461-1221 (ext. 3200)

\begin{abstract}
This research presents a solution to the problem of planning the optimum area for economic crops by developed mathematical models and developed an algorithm to solve the problem of planning the optimum area by considered economic value for the maximize profit of farmers. The data were collected from farmers in 8 provinces in the northeastern region of Thailand. The 3 economic crops studied were rice, cassava and sugarcane. The solving problem methods were 1) Created mathematical models and solved the problems with Lingo V.11. 2) Improved Differential Evolution algorithms (I-DE) to solve the problems, which had 3 local search methods included (Swap, Cyclic Move and K-variable moves). The results of this study showed that in the small and medium problems instances, Lingo V.11 and DE provided equal profit outcome but DE was faster but in the large size of test instances DE generated better solution than that of Lingo v.11 when Lingo simulation time is set to 250 hours and DE simulation time has set to maximum 21.82 minutes. 2) Comparing DE and I-DE , I-DE outperforms DE in finding the better solution for all size of test instances (small, medium and large).
\end{abstract}

Keywords: Differential Evolution Algorithm; Crop Planning; Economic Crops; Improvement Differential Evolution algorithm

\section{Introduction}

There are variety of crops planting in Thailand which is different in each part of the country, as Thailand has many important world-class economic crops; rice, cassava, sugarcane, rubber, palm and so on. These crops are planted in different parts, belong to these factors; land, soil, water and weather, since Thailand has many facilitation factors for the growth of them. In choosing crops, Thai farmers usually determine from these basic factors such as land, soil, water and weather which most of areas in Thailand are very suitable for planting crops [1] Currently, Thai economic crops play an important role in the economic growth of the country which has very high value. Therefore, the crop planting in northeastern region of Thailand is 63.84 million rai or approximately 23.27 rai per household [2]. From this information, it can be summarized that north east of Thailand has very high numbers of planting crops. While there are high numbers of supply but unbalance with demand, this causes 
variety of problems as follow: rice farmers invaded Bangkok to protest as delayed payment in rice subsidy program and sugarcane farmers and Para rubber farmers closed a main road to protest over low prices. The main problems of the exceed demand are include, unsuitable crop planting, high investment - low profit, unbalanced demand and supply, poor plantation knowledge, unsuitable marketing, and inconvenient transportation. These problems need to be considered the factors related to crop planning; the allotment for each crop planning, demand and supply, cost and profit, marketing, transportation distance and so on.

There are several factors related to crop planning include, demand and supply, planting areas, profit, weather condition and others, which is difficult for farmers to analyze by themselves, due to equipment and knowledge limitation. These factors cause farmers to not be able to know the suitable or limit of their planting areas. Therefore, the researchers wanted to solve the crop planning problems for farmers in the lower northeast region of Thailand, by developing effective mathematical models and algorithms, considered economic value, so farmers would gain higher profit from suitable crop planning. From the result farmer would be able to plan suitable planting areas for the 3 crops, increase production and decrease transportation distance and plant proper crops.

\section{Literature Review}

Differential Evolution algorithm (DE) was developed from Evolution Strategy (ES) by Price and Storn [3]. DE was used to enhance performance and reliable of operations which also could be applied in finding answers for simple operations. DE was first published about technical report in 1995. Furthermore, using DE to solve logistics problems also attracted some researchers and the program was developed in 2009, Erbao and Mingyong [4] used DE to solve vehicles route problems (VRP) and in Fuzzy demands, it was used as conditions to design model of the program in stochastic simulation and to create algorithm of Hybrid intelligent. The result of the study was; index of dispatcher settings got the best value by using crossover parameter in developing different answers from local optimum. Later, Dechampai D. et al [5] presented DE Algorithm in VRP problem which had limit of vehicle capacity in poultry industry, by using 2 heuristics; DE_G-Q-DVRP-FD and MSEOMDE_G-Q-DVRP-FD. Both heuristics were used for grouping customers before arranging transportation routes, which provided lower cost than the present method using at 7.59-31.28\% and better than the $1^{\text {st }}$ Heuristic at $0.84-13.15 \%$. A year later, Sethanan and Pitakaso [6] presented DE for scheduling of raw milk transportation The purpose were to lowest the cost in the fuel cost, cleaning cost and disinfection of raw milk tank in vehicle, by presenting modification of 5 DE methods and 2 steps of emerging and survival process which was called re-born vector. The results were shorter routes and less trucks transportation besides, in the same year, Sethanan and Pitakaso [7] presented the development of DE in solving general operations by using 3 Local Search techniques to find better answers. Those 3 techniques were developed into 7 other methods. Besides, they were also measured the effectiveness of each method to find the best way to compare with BEE algorithm and Tabu algorithm in the experiment set of Gapa-Gape, result of DE-SK was better than another 2 methods.

For the literature review, which the researcher had studied some studied related to Differential Evolution for crop planning are as follow; Crop planning problem [8] Studied plant production pattern [9] Finding cropping patterns focused on the selection of crops and the allocation of cultivated areas using DE and Gradient Based Methods [10], [11]. Using DE to solve Crew Rostering problems by Santosa B. et al. [12]. The use of DE for multi-objective crop planning [13]. The Use of Ensemble of Parameters Strategies of Differential Evolution Algorithm (EPSDE) by Parameters and Mutation and Crossover [14]. Development of multi-objective algorithms for optimal crop planning [15] and using DE for crop planning single and multi-objective optimization model [16]. For Pant, M. et al. [17] 
presents an application of Differential Evolution (DE) to determine optimal crop plan for command area of Pamba-Achankovil-Vaippar (PAV) link project. And Yi H. et al. [18] used three improved hybrid metaheuristic algorithms for engineering design optimization. From these studies above could be summarized that $\mathrm{DE}$ is a very effective algorithm to find the answers, this was the important part for the researcher's decision.

\section{Mathematical Model for the Crop Planning}

In planting the 3 economic crops, each farmer was assigned to plant only 1 crop and each farmer was allocated a transport vehicle by considering the lowest transport costs to maximize profit, considering the most economical value. The products of each farmer were sent to the factory or purchased site according to the type of crop, as figure 1.

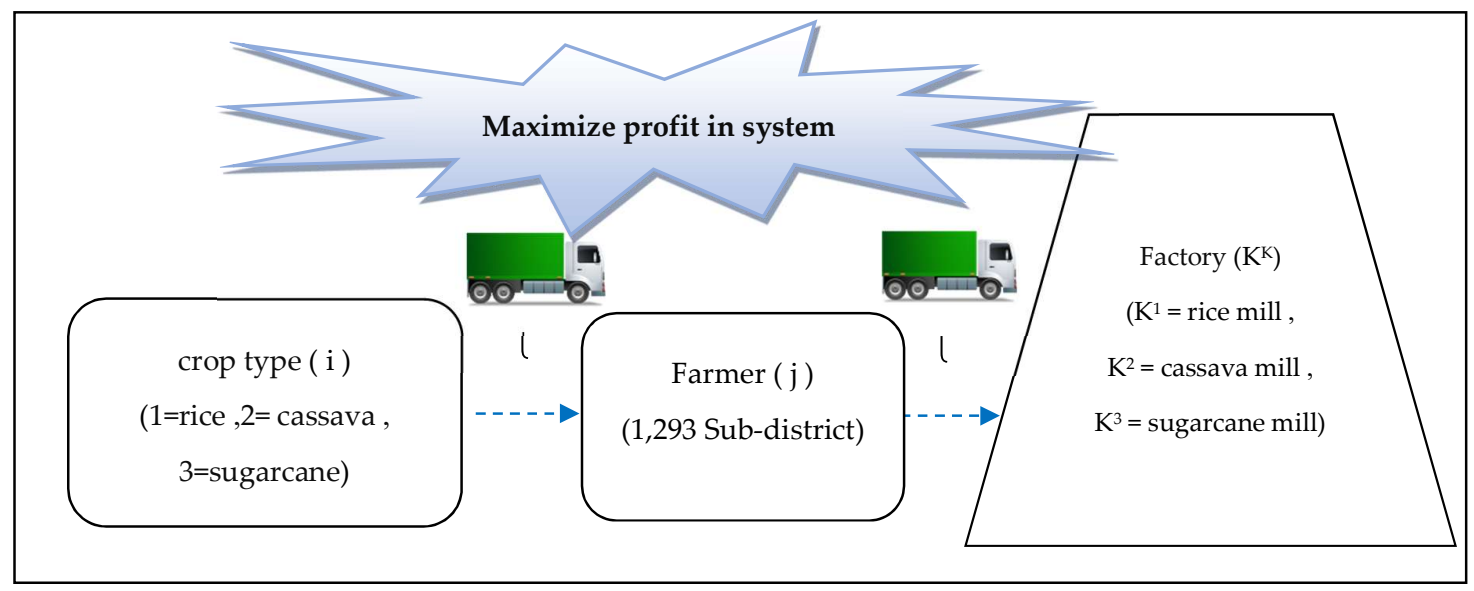

Figure 1. Model Transport of economic crops from the farmer to the factory.

The mathematical model for the economic crop planning were as follows

\subsection{Index:}

i stands for crop type ( $1=$ rice, $2=$ cassava, $3=$ sugarcane)

$\mathrm{j} \quad$ stands for planning areas $(\mathrm{j}=1,2, \ldots, \mathrm{J})$

$\mathrm{K}$ stands for factory $\left(\mathrm{K}=1 \ldots \ldots \mathrm{K}^{\mathrm{i}}\right.$, when $\mathrm{K}^{1}=$ rice mill, $\mathrm{K}^{2}=$ cassava mill, $\mathrm{K}^{3}=$ sugarcane mill)

\subsection{Parameters:}

$\mathrm{P}_{\mathrm{ij}} \quad$ stands for crop price i planted by farmer $\mathrm{j}$ (Baht/Kilogram)

$\mathrm{C}^{1_{\mathrm{ij}}} \quad$ stands for cost of planning i planted by farmer $\mathrm{j}$ (Baht/Rai)

$B_{i j} \quad$ stands for rate of crop yield i planted by farmer $j$ (Ton/Rai)

$\mathrm{A}_{j} \quad$ stands for planning area in each district (Rai)

$D_{\mathrm{jk}} \quad$ stands for distance from planning area $\mathrm{j}$ to factory $\mathrm{k}$ (kilometer)

$\mathrm{C}^{2}{ }_{\mathrm{i}} \quad$ stands for transportation costs of each crop $\mathrm{k}$ (Baht/Kilometer)

$\mathrm{CK}_{\mathrm{K}} \quad$ stands for factory purchase capacity (Ton)

$\mathrm{C}^{3 \mathrm{ij}} \quad$ stands for cost of crop cultivation i planted by farmer $\mathrm{j}$ (Baht/Rai)

$\mathrm{V}_{j}$ stands for transportation capacity (Ton)

$\mathrm{M}$ stands for maximum production capacity 


\subsection{Decision Variables}

$$
\begin{aligned}
& \mathrm{X}_{\mathrm{ijk}}=\left\{\begin{array}{l}
1=\text { if there is transportation } \mathrm{i} \text { from farmer } \mathrm{j} \text { to factory } \mathrm{k} \\
0=\text { other cases }
\end{array}\right. \\
& \mathrm{Y}_{\mathrm{ij}} \\
& \mathrm{H}_{\mathrm{ijk}}=\text { Quantity which factory } \mathrm{k} \text { is given from crop } \mathrm{i} \text { from farmer } \mathrm{j} \\
& \mathrm{T}_{\mathrm{ij}}=\text { Number of transport cycles must be integer (Round) } \\
& \mathrm{T}^{2} \mathrm{ij}=\text { Number of crop transportation } \mathrm{i} \text { by farmer } \mathrm{j} \text { (Round) }
\end{aligned}
$$

\subsection{Mathematical Model}

The researchers designed and developed the mathematical model by considering the maximize profit for farmers. The related factors were considered consists of crop price, cost of each crop cultivation, yield rate of each crop, planning area in each district, transportation distance from planning area to factory, cost of each crop transportation, amount of each crop transportation and cost of cultivation were as follows:

\section{Objective Function}

$\operatorname{Maximize} \mathbf{Z}=\sum_{i=1}^{I} \sum_{j=1}^{J}\left(P_{i j}-C^{1}{ }_{i j}\right) Y_{i j} B_{i j} A_{j}-\sum_{i=1}^{I} \sum_{j=1}^{J} \sum_{k=1}^{K} X_{i j k} D_{j k} C^{2}{ }_{i} T^{2} i j-\sum_{i=1}^{I} \sum_{j=1}^{J} Y_{i j} C^{3}{ }_{i j}$

The objective function, focused on economics for the maximize profit of farmers. In Formula (1) consisted of 3 main sequences as follows. Sequence 1) Function of raw material cost which depended on the purchase price of each crop, cost of planting each crop, size of planting area and yield rate. Sequence 2) Function of transportation cost which depended on the amount of raw material, transportation distance to factory, cost of transportation for each crop and amount of transportation round of each crop. And sequence 3) Function of cost of raising crop which depended on budget in raising each crop.

\subsection{Constraints}

$$
\sum_{i=1}^{I} Y_{i j}=1
$$

For the constraints function consisted of (2) Formula of limit that each farmer can only plant one crop. 
$\sum_{j=1}^{J} Y_{i j} \geq 1$

$\forall{ }_{i \in I}$

For Formula (3) of limit that each crop requires at least one farmer.

$$
\sum_{K \in \mathrm{K}^{\mathrm{i}}} H_{i j k}=Y_{i j} \cdot B_{i j} \cdot A_{j} \quad \forall_{i \in I} \forall_{j \in J}
$$

For Formula (4) Functions of limit that the amount of crop yield to be delivered to the factory must be equal to the amount of crop planted by each farmer.

$$
H_{i j k} \leq M . X_{i j k} \quad \forall_{i \in I} \forall_{j \in J} \forall_{k \in K}
$$

For Formula (5) of limit that the amount of crop yield to be delivered to the factory must not exceed the amount of crop planted by each farmer.

$$
\sum_{k=1}^{K} X_{i j k} \leq 1 \quad \forall \forall_{i \in I} \forall_{j \in J}
$$

For Formula (6) of limit that a farmer can only transport only one route to the factory.

$$
\sum_{j=1}^{J} H_{i j k} \leq C_{K} \quad \forall_{i \in I} \forall_{K \in K^{i}}
$$

For Formula (7) of limit that the amount of crop must not exceed the purchase capacity of the factory.

$$
T^{2}{ }_{i j} \geq T^{1}{ }_{i j} \quad \forall_{i \in I} \forall_{j \in J}
$$

For Formula (8) of limit that the amount of transportation round must come from yield rate multiplied by the crop area and divided by the capacity of the transport vehicle.

$$
T^{1}{ }_{i j}=\frac{Y_{i j} \cdot B_{i j} \cdot A_{j}}{V_{j}} \quad \forall_{i \in I} \forall_{j \in J}
$$

For Formula (9) of limit that the amount of transportation round must be integer (round).

$$
Y_{i j} \cdot M \geq \sum_{K \in K^{i}}^{K} X_{i j k} \quad \forall_{i \in I} \forall_{j \in J}
$$

For Formula (10) of limit that the maximum yield rate delivered when a farmer is assigned crop planting must not be less than the amount of yield rate send from the farmer to the factory.

$$
Y_{i j} \text { (bin) } \quad \forall_{i \in I} \forall_{j \in J}
$$

For Formula (11) of limit that farmer who plants each crop, value 0 or 1 only. 1 for plant and 0 for others.

$$
X_{i j k} \text { (bin) } \quad \forall_{i \in I} \forall_{j \in J} \forall_{k \in K}
$$


For Formula (12) Decision Variables when a farmer $\mathrm{j}$ transport each crop to the factory $\mathrm{k}$, value 0 or 1 only.

$$
H_{i j k}(\text { gin }) \quad \forall_{i \in I} \forall_{j \in J} \forall_{k \in K}
$$

For Formula (13) of limit that the amount of crop i from farmer $\mathrm{j}$ to the factory $\mathrm{k}$, value is an integer.

$$
T_{i j}^{2}(\text { gin }) \quad \forall_{i \in I} \forall_{j \in J}
$$

And for Formula (14) Formula of limit that the amount of transportation round is an integer.

\section{Original Differential Evolution Algorithm}

The researcher used Differential Evolution algorithm (DE) to find the outcome [3], for solving crop planning's problems. There were 4 steps as follow.

4.1 Initial population; a process of random sample selection from the population under a certain limit which could be set or NP: Number of population. The group of population was calculated for the answer. It was called Fitness Value: the process of creating the initial answer, using the initial population to create the answer.

4.2 Mutation Process; The process of multiplying the decision variable by a factor called the Weighting Factor: F or called Mutation Factor: F For the purpose of mutation to get new different answers from the initial population. There are steps as follows

4.2.1 Set Target Vector $(\mathrm{Xi}, \mathrm{G}) \mathrm{i}=1,2,3, \ldots, \mathrm{NP}$

4.2.2 Random number selection 3 Vector $\left(X_{r} 1, G, X r 2, G, X r 3, G\right)$ from the initial population which different from Target Vector

4.2.3 Calculate for Mutant Vector (Vi,G+1) by Formula (15)

$\mathrm{Vi}, \mathrm{G}+1=\mathrm{Xr1}, \mathrm{G}+\mathrm{F}(\mathrm{Xr2}, \mathrm{G}-\mathrm{Xr3}, \mathrm{G})$

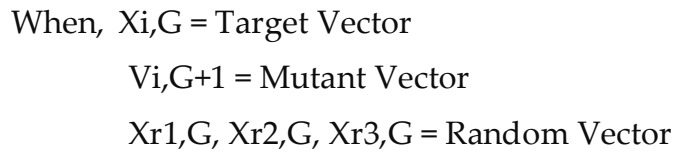

For $\mathrm{F}$ is Weighting Factor value 0 to 2, and set $\mathrm{F}=2.0$ [7], [19]. Change value in coordinate of vector by using random numbers to change the value and into the mutation process (Mutant vector).

4.3 Crossover or Recombination Process; It was mixed species process which produced new species of better or worse result for selection of decision variables. The result was Trial Vector (Ui,G+1) Set $\mathrm{Cr}=0.8$ [7], [19], then enter value exchange by Formula (16),

$$
\mathrm{U}_{\mathrm{i}, \mathrm{G}+1}=\left\{\begin{array}{l}
\left.\mathrm{V}_{\mathrm{ij}}, \mathrm{G}+1 \text { if } \text { Rand }_{\mathrm{ij}} \leq \mathrm{CR}\right) \text { or } \mathrm{j}=\text { Irand } \\
\left.\mathrm{X}_{\mathrm{ij}, \mathrm{G}} \text { if } \text { Rand }_{\mathrm{ij}}>\mathrm{CR}\right) \text { or } \mathrm{j} \neq \text { Irand }
\end{array}\right.
$$


4.4 Selection Process; It was the selection process for the best answer between Target Vector and Trial Vector using Formula (17) by compare Function Value or Cost Value of Trial Vector with Target Vector. In case of Function Value of Trial Vector was better than Target Vector would be replaced by Trial Vector in the next generation.

$$
X_{i, j, G+1}=\left\{\begin{array}{c}
U_{i, j, G} \text { if }\left(U_{i, j, G}\right) \leq f\left(X_{i, j, G}\right) \\
X_{i, j, G} \text { if otherwise }
\end{array}\right.
$$

Then adjust the answers in each NP for a chance of a better answer. Found out the answer (objective) from calculation, compared and choose the best answer from all the population

\section{Improved Differential Evolution Algorithm}

This topic is about developing and improving algorithms by DE, using Dev-C++ for testing on 3 different sizes of problems. Researcher used DE with Local Search to develop algorithm, by adding a specific search step, after the value exchange process in the recombination. By algorithms were developed to provide better results. There were 3 methods; 1 ) Swap Algorithm, adapted from method of Diaz \& Fernandez, [20]. 2) Cyclic Move Algorithm. 3) K-Variable Move Algorithm, for step improved DE see in figure 2.

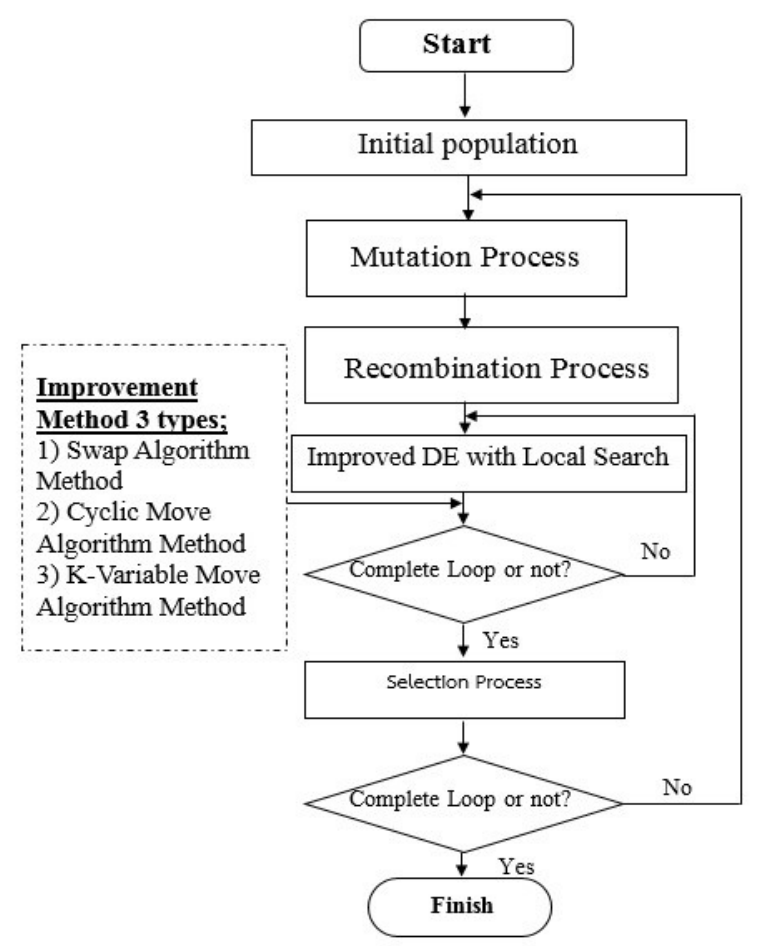

Figure 2. Step using improve DE with Local Search 3 methods.

5.1 Swap Algorithm A method to improve the Heuristic-based solution by switching pair positions between groups of members. Assuming, originally assigned farmer $10^{\text {th }}$ to plant rice, algorithm will switch with the farmer $24^{\text {th }}$ who planted cassava, to make more profit. Then switch the farmer $8^{\text {th }}$ who planted cassava with the farmer $30^{\text {th }}$ who planted sugarcane, relocated all the position in the same way but the amount of output that the farmer sells must not exceed the capacity of the 
factory. In switching positions, consider switching pairs that make more profit than before, see in figure 3 .

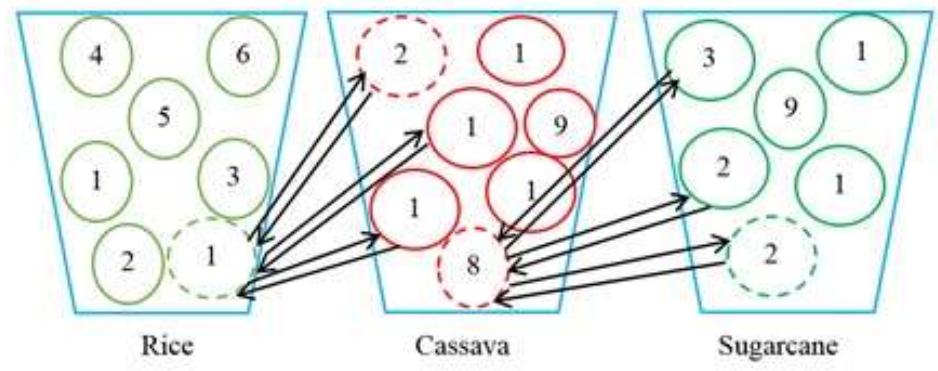

Figure 3. An example to develop answer by Swap Method.

5.2 Cyclic Move Algorithm This method selected 1 famer from each group then switched each farmer in circle. For example; originally assigned to the farmer $6^{\text {th }}$ which planted rice, changed to cassava. Next, the farmer $8^{\text {th }}$ which planted, changed to sugarcane. And the farmer $11^{\text {th }}$ which planted sugarcane, changed to rice, relocated all the position in the same way by random all rounds. In switching positions, consider switching pairs that make more profit than before, as figure 4 .

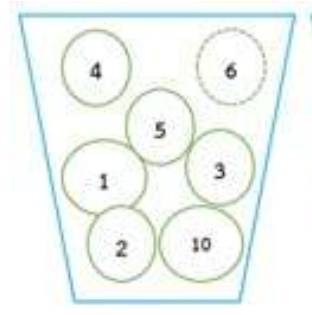

Rice

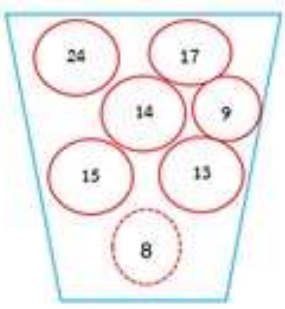

Cassava

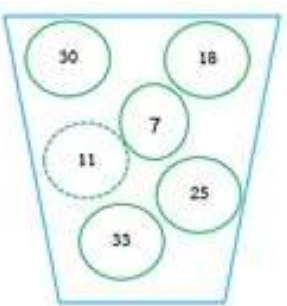

Sugarcane

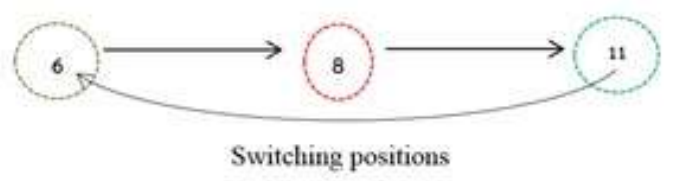

Figure 4. An example to develop answer by Cyclic Move Method.

5.3 K-Variable Move Algorithm By set $\mathrm{K}=5$ (from randomly testing by random all rounds) then chose 1 crop from each group, switched the farmer to another crop. For example; originally assigned the farmer $17^{\text {th }}$ to planted cassava, changed to sugarcane. Next the farmer $33^{\text {rd }}$ which planted sugarcane, changed to cassava. The farmer $8^{\text {th }}$ which planted cassava, changed to sugarcane. The farmer $24^{\text {th }}$ which planted sugarcane, changed to rice. And the farmer $1^{\text {st }}$ which planted rice, changed to cassava. All the position relocated in the same way but the amount of output that the farmers sell, must not exceed the capacity of the factory. In switching positions, consider switching pairs that make more profit than before, as figure 5 . 


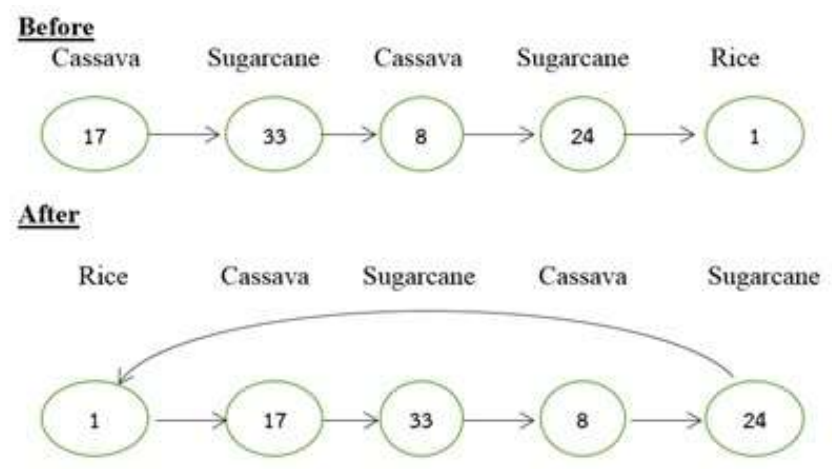

Figure 5. An example to develop answer by K-Variable Move Method.

\section{Computational Experiment and Results}

The computational result is divided into two parts. First part, the result of the proposed method (DE) comparing with result generated by Lingo V.11 is presented to check if our proposed heuristics is reliable and trustable. The second part of the simulation is used to check if the improved DE (I$\mathrm{DE}$ ) is better than that of the original DE so that the contribution of adding three local search methods to the original DE has benefit to the original algorithm.

\subsection{Experimental results of DE compared with Lingo v.11}

The researchers used General DE and developed DE to apply and solved problems by using Dev-C++ in design algorithms and calculate to compare the outcome with the processing unit; Intel (R) CoreTM i5-2410M 2.3 GHz and 4 GB memory. DE is run 5 replicates and the best solution among all five runs is the solution represented in Table 1. The problems instances were categorized into 3 groups; 1) small problems group, 5-20 farmers 2) medium problems group, 40-70 farmers. And 3) Large problems group and 80-500 farmers for the case studies' problems. There are 4 test instances randomly generated for small size of instances while there are 3 test instances generated in medium and large size of test instances. 1 real case study is used in the simulation test. In total we have 11 test instances. The stopping criteria for lingo is set to maximum 250 hours or when it find the optimal solution. The stopping criteria of DE is number of iteration which is 500 iterations. Set NP=50, F = 2.0, $\mathrm{CR}=0.8$ [7], [19]. Result of DE comparing with Lingo v.11 is shown in Table 1 .

Table 1. Experimental results with DE compared with Lingo V.11

\begin{tabular}{|c|c|c|c|c|c|c|c|c|}
\hline \multirow{3}{*}{$\begin{array}{l}\text { Proble } \\
\text { m } \\
\text { Group }\end{array}$} & \multirow{3}{*}{$\begin{array}{c}\text { Farmer } \\
\text { or } \\
\text { Sub- } \\
\text { district }\end{array}$} & \multirow{3}{*}{$\begin{array}{c}\text { Rice } \\
\text { Number } \\
\text { of } \\
\text { factories }\end{array}$} & \multirow{3}{*}{$\begin{array}{c}\text { Cassava } \\
\text { Number } \\
\text { of } \\
\text { factories }\end{array}$} & \multirow{3}{*}{$\begin{array}{c}\text { Sugarca } \\
\text { ne }\end{array}$} & \multicolumn{4}{|c|}{ Methods } \\
\hline & & & & & \multicolumn{2}{|c|}{ Lingo } & \multicolumn{2}{|c|}{ DE } \\
\hline & & & & & $\begin{array}{c}\text { Solution } \\
\text { (Baht) }\end{array}$ & $\begin{array}{c}\text { Time } \\
\text { (Second) }\end{array}$ & Solution & $\begin{array}{c}\text { Time } \\
\text { (Second) }\end{array}$ \\
\hline 1 & 5 & 3 & 3 & 4 & 153,040 & 00:00:02 & 153,040 & 00:00:01 \\
\hline (Small & 10 & 3 & 3 & 4 & 538,909 & 00:00:05 & 538,909 & 00:00:03 \\
\hline \multirow[t]{2}{*}{ Size) } & 15 & 3 & 3 & 4 & 704,463 & 00:00:27 & 704,463 & 00:00:05 \\
\hline & 20 & 7 & 7 & 5 & $1.1175 \times 10^{6}$ & 00:00:46 & $1.1175 \times 10^{6}$ & 00:00:14 \\
\hline
\end{tabular}




\begin{tabular}{ccccccccc}
\hline 2 & 40 & 16 & 30 & 5 & $2.4585 \times 10^{6}$ & $00: 03: 39$ & $2.4585 \times 10^{6}$ & $00: 00: 23$ \\
(Medi & 60 & 30 & 50 & 5 & $3.73617 \times 10^{6}$ & $00: 06: 50$ & $3.73617 \times 10^{6}$ & $00: 01: 38$ \\
um & 70 & 35 & 55 & 3 & $4.31865 \times 10^{6}$ & $00: 10: 25$ & $4.31865 \times 10^{6}$ & $00: 03: 37$ \\
size) & & & & & & & & \\
\hline 3 & 80 & 45 & 60 & 5 & $4.3341 \times 10^{6}$ & $250 \mathrm{Hrs}^{*}$ & $4.45924 \times 10^{6}$ & $00: 02: 13$ \\
$($ Large & 100 & 60 & 80 & 10 & $4.4355 \times 10^{6}$ & $250 \mathrm{Hrs}^{*}$ & $4.51473 \times 10^{6}$ & $00: 13: 10$ \\
Size) & 500 & 70 & 85 & 13 & $5.13148 \times 10^{6}$ & $250 \mathrm{Hrs}^{*}$ & $5.18148 \times 10^{6}$ & $00: 16: 34$ \\
\hline Case & 1293 & 95 & 98 & 19 & $13.9234 \times 10^{6}$ & $250 \mathrm{Hrs}^{*}$ & $14.1761 \times 10^{6}$ & 00.21 .82 \\
study & & & & & & & & \\
\hline
\end{tabular}

Remarks * Best solution generated in 250 hours.

Resulting from table 1, in small and medium size of test instances DE can find the same solution of Lingo v.11 when using lower computational time. In the large size of instances (including case study), DE generates better solution that that of Lingo v.11 that using 250 hours run while DE uses maximum 21.82 minutes to obtain the result. In all test instances, DE uses less computational time than that of DE.

\subsection{Experimental results with DE compared with Modified DE}

We have 3 improved DE algorithms (I-DE) which are I-DE-SW (DE + SWAP) , I-DE-CY (DE+ Cyclic move) and I-DE-KV (K-variable moves). The stopping criteria using in this session is simulation times which is set to 2 hours. 5 times of the simulation run are performed and the best solution among all runs is shown in table 2.

Table 2. Overall profitability for the general DE and I-DE method at equal duration

\begin{tabular}{|c|c|c|c|c|c|}
\hline \multirow{2}{*}{ Problem size } & \multirow{2}{*}{$\begin{array}{c}\mathrm{N} \\
(\mathrm{A}-\mathrm{B}-\mathrm{C}) \\
\end{array}$} & \multicolumn{4}{|c|}{ Solution (Profit : Baht) } \\
\hline & & DE & I-DE-SW & I-DE-CY & I-DE-KV \\
\hline \multirow{8}{*}{$\begin{array}{l}\text { Small } \\
\text { size }\end{array}$} & 5 & 153,040 & 208,933 & 153,040 & 208,933 \\
\hline & $(3-3-4)$ & & & & \\
\hline & 10 & 538,909 & 565,109 & 554,117 & 565,109 \\
\hline & $(3-3-4)$ & & & & \\
\hline & 15 & 704,463 & 704,463 & 495,322 & 704,219 \\
\hline & $(3-3-4)$ & & & & \\
\hline & 20 & $1.1175 \times 10^{6}$ & $1.10857 \times 10^{6}$ & $1.11875 \times 10^{6}$ & $1.11877 \times 10^{6}$ \\
\hline & $(7-7-5)$ & & & & \\
\hline \multirow{6}{*}{$\begin{array}{l}\text { Medium } \\
\text { size }\end{array}$} & 40 & $2.4585 \times 10^{6}$ & $2.4585 \times 10^{6}$ & $2.4585 \times 10^{6}$ & $2.4585 \times 10^{6}$ \\
\hline & $(16-30-5)$ & & & & \\
\hline & 60 & $3.73617 \times 10^{6}$ & $3.73617 \times 10^{6}$ & $3.73617 \times 10^{6}$ & $3.73711 \times 10^{6}$ \\
\hline & $(30-50-5)$ & & & & \\
\hline & 70 & $4.31865 \times 10^{6}$ & $4.31865 \times 10^{6}$ & $4.31865 \times 10^{6}$ & $4.31865 \times 10^{6}$ \\
\hline & $(35-55-3)$ & & & & \\
\hline \multirow{4}{*}{$\begin{array}{l}\text { Large } \\
\text { size }\end{array}$} & 80 & $4.55924 \times 10^{6}$ & $4.55924 \times 10^{6}$ & $4.55924 \times 10^{6}$ & $4.56032 \times 10^{6}$ \\
\hline & $(45-60-5)$ & & & & \\
\hline & 100 & $4.65924 \times 10^{6}$ & $4.71105 \times 10^{6}$ & $4.65924 \times 10^{6}$ & $4.71105 \times 10^{6}$ \\
\hline & $(60-80-10)$ & & & & \\
\hline
\end{tabular}




\begin{tabular}{cccccc} 
& 500 & $5.23148 \times 10^{6}$ & $5.37866 \times 10^{6}$ & $5.23148 \times 10^{6}$ & $5.37866 \times 10^{6}$ \\
& $(70-85-13)$ & & & & \\
\hline Case & 1,293 & $14.27614 \times 10^{6}$ & $14.57129 \times 10^{6}$ & $14.32278 \times 10^{6}$ & $14.59643 \times 10^{6}$ \\
studies & $(95-98-19)$ & & & & \\
\hline
\end{tabular}

Remark; N (A-B-C) means N stands for Farmers, A stands for rice mill, B stands for cassava mill, and C stands for sugarcane mill.

From results in Table 2, the experiment to compare the efficiency of original DE and Improved DE (I-DE) using the same duration (2 hours). I-DE-KV provided the most effective answer and most comprehensive range of answer than other methods, especially for large problems and case studies for 1,293 farmers, which the best answer of the case studies found from DE-KV was 14,596,430 baht per Crop cycle and also could show the factory location and areas for economic crop planning as well.

\section{Conclusions}

This study aims to solve the allotment of economic crop planning for farmers in 8 provinces in the lower northeast region of Thailand by improving mathematical models and algorithms, considered economic value for the maximize profit of farmers. Focused on 3 economic crops; rice, cassava and sugarcane. The researcher used Differential Evolution algorithm (DE) to solve the problems for economics purpose; the maximize profit of farmers. The outcomes and time durations of DE algorithms were compared with Lingo V.11. When compared the performance of all algorithms using DE in small problems simulation, DE had the best performance and also enhanced chance in finding better outcome.

There are three algorithms proposed in this article which are I-DE-SW (DE + SWAP) , I-DE-CY (DE+ Cyclic move) and I-DE-KV (K-variable moves). From the computational result, I-DE-KV is the best proposed method comparing with all proposed methods including the original DE algorithm. All improve DE (I-DE) can find better solution than that of the original DE. It can conclude that DE which include the local search can improve the efficiency of the original version of it. The SWAP limits number of entities involved to 2, while cyclic move has 3 entities involved and K-cyclic move can have 2,3,4 or more depending on random value of $\mathrm{K}$. This attributes make $\mathrm{K}$-variable moves are more free and flexible thus it can generated the best solution among all proposed heuristics while using the same computational time.

Acknowledgments: We would like to thank Metaheuristics for Logistics Optimization Laboratory, Department of Industrial Engineering, Department of Industrial Engineering, Faculty of Engineering, Khon Kaen University and Department of Economics, Faculty of Business Administration, Rajamangala University of Technology Thanyaburi for funding this research.

Author Contributions: U.K. and R.P. collect data, design algorithm. T.S. performs the experiment while K.S analysis the result.

Conflicts of Interest: The authors declare no conflicts of interest.

\section{References}

1. Chumroen Yuenyongsawat, et al. Principles of agriculture, Department of Plant Science, Faculty of natural resources, Prince of Songkla university, Had Yai campus, Songkhla, Thailand, 2000.

2. Agricultural Information Office. Agricultural Information, Agricultural Economics, Ministry of Agriculture and Cooperatives, Bangkok, Thailand, 2016. 
3. Storn, R.; Price, K. V. “Differential evolution: a simple and efficient adaptive scheme for global optimization over continuous spaces. Journal of Global Optimization. 1995, 11, 341-359, DOI: 10.1023/A:1008202821328

4. Erbao, C.; Lai, M. A Hybrid differential evolution algorithm to vehicle routing problem with fuzzy demands. Journal of Computational and Applied Mathematics. 2009, 231, 302-310, DOI: ttps://doi.org/10.1016/j.cam.2009.02.015

5. Dechampai, D.; Tanwannichkul, L.; Sethanan, K.; Pitakaso, R. A differential evolution algorithm for the capacitated VRP with Flexibility for mixing pickup and delivery services and the maximum duration route in poultry industry. Journal of Intelligent Manufacturing. 2017, 28, 1357-1376, DOI: https://doi.org/10.1007/s10845-015-1055-3

6. Sethanan, K.; Pitakaso, R. Differential evolution algorithms for scheduling raw milk transportation. Computers and Electronics in Agriculture. 2016a, 121, 245-259, DOI:

https://doi.org/10.1016/j.compag.2015.12.021

7. Sethanan, K.; Pitakaso, R. Improved differential evolution algorithms for solving generalized assignment problem. Expert Systems with Applications. 2016b, 45, 450-459, DOI: https://doi.org/10.1016/j.eswa.2015.10.009

8. Das, S.; Suganthan, P.N. Differential Evolution: A Survey of the State-of-the-Art. IEEE Transactions on Evolutionary Computation, 2011, 15, 4-31, DOI: 10.1109/TEVC.2010.2059031

9. Glen, J. J. Mathematical models in farm planning: A survey. Operations Research. 1987, 35, 641-666, DOI: https://doi.org/10.1287/opre.35.5.641

10. Itoh, T.; Ishii, H.; Nanseki, T. A model of crop planning under uncertainty in agricultural management. International Journal of Production Economics. 2003, 81, 555-558.

DOI: https://doi.org/10.1016/S0925-5273(02)00283-9

11. Sarker, Talukdar, S.; Haque, A. Determination of optimum crop mix for crop cultivation in Bangladesh. Applied Mathematical Modelling. 1997, 21, 621-632, DOI: https://doi.org/10.1016/S0307-904X(97)00083-8

12. Santosa, B.; Sunarto, A.; Rahman, A. Using Differential Evolution Method to Solve Crew Rostering Problem. Applied Mathematics. 2010, 1, 316-325, DOI: 10.4236/am.2010.14042

13. Adeyemo, J.; Otieno, F. Differential evolution algorithm for solving multi-objective crop planning model. Agricultural Water Management. 2010, 97, 848-856, DOI: https://doi.org/10.1016/j.agwat.2010.01.013

14. Mallipeddi, R.; Suganthan, P. N.; Pan, Q. K.; Tasgetiren, M. F. Differential evolution algorithm with ensemble of parameters and mutation strategies. Applied Soft Computing, 2010, 11, 1679-1696.

DOI: https://doi.org/10.1016/j.asoc.2010.04.024

15. Oluwole, A.; Adekanmbi,; Oludayo O.; Olugbara, Adeyemo, J. A Comparative Study of State-of-the-Art Evolutionary Multi-objective Algorithms for Optimal Crop-mix planning. International Journal of Agricultural Science and Technology. 2014, 2, 8-16, DOI: 10.14355/ijast.2014.0301.02.

16. Adeyemo, J.; Bux, F.; Otieno, F. Differential evolution algorithm for crop planning: Single and multiobjective optimization model. International Journal of the Physical Sciences. 2010, 5, 1592-1599. DOI: $10.5897 / \mathrm{IJPS}$

17. Pant, M.; Radha, T.; Rani, D.; Abraham, A.; Srivastava, D. K. Estimation Using Differential Evolution for Optimal Crop Plan. Hybrid Artificial Intelligence Systems. HAIS 2008. Lecture Notes in Computer Science. 2008, 5271, 289-297. DOI: https://doi.org/10.1007/978-3-540-87656-4_36

18. Yi, H.; Duan, Q.; Liao. T. W. Three improved hybrid metaheuristic algorithms for engineering design optimization. Applied Soft Computing. 2013, 13, 2433-2444. DOI: 13 (2013) 2433-2444

19. Qin, A. K.; Huang, V. L.; Suganthan, P. N. Differential evolution algorithm with strategy adaptation for global numerical optimization. IEEE Transactions on Evolutionary Computation. 2009, 13, 398-417. Retrieved from http://ieeexplore.ieee.org/document/4632146/.

20. Diaz, J.A.; Fernandez, E. A Tabu search heuristic for the generalized assignment problem. European Journal of Operational Research. 2001, 132, 22-38, DOI: https://doi.org/10.1016/S0377-2217(00)00108-9 